\title{
The Predictive Effects of Online Communication on Well-Being among Chinese Adolescents
}

\author{
Jinliang Wang ${ }^{1}$, Haizhen Wang ${ }^{2}$ \\ ${ }^{1}$ Southwestern University, Chongqing, China; \\ ${ }^{2}$ Chongqing Youth Vocational and Technical College, Chongqing, China. \\ Email: WJL200789@163.com, haizhen@swu.edu.cn \\ Received March $7^{\text {th }}, 2011$; revised April 18 $8^{\text {th }}, 2011$; accepted May 22 $2^{\text {nd }}, 2011$.
}

\begin{abstract}
Based on theories and previous studies on online communication, we proposed two hypotheses to better understand the effects of online communication on subjective well-being with a sample of Chinese adolescents $(n=$ 190). The results of liner regression analysis found that online communication was positively related to adolescents' subjective well-being. For boys, the coefficient of determination of online communication on subjective well-being was .143, and for girls the coefficient of determination was .040 , indicating that boys benefit more from online communication than do girls.
\end{abstract}

Keywords: Online Communication, Subjective Well-Being, Adolescent

\section{Introduction}

Internet has significantly changed our way of living, communicating, and information seeking in the last decade. As a defining Internet user group, adolescents are more apt to online communication because of their developmental stage. According to an investigation by China Internet Network Information Center (CINIC) (2009), there are 384 million Internet users in China, $50.7 \%$ of which are adolescents (below 25 years). However, it is surprising that researchers have not given enough attention to such a large Internet user group, with only a few studies having been conducted on Chinese adolescents' online communication. Online communication, such as Instant Messaging, Internet-based chat-room, and social network sites, has been suggested to have obvious impact on users' subjective well-being, especially for adolescents due to their curiosity about new emerging technologies and their developmental stage.

Many researchers have examined the association between adolescents' online communication and subjective well-being (Carden \& Rettew, 2006; Schiffrin, Edelman, Falkenstern, \& Stewart, 2010; Valkenburg \& Peter, 2007). However, so far no consistent conclusions have been made. A review on literature reveals that at least two theoretical perspectives exist regarding the effects of online communication on users' well-being: the displacement hypothesis and the augmentation hypothesis. The displacement hypothesis asserts that time spent using a given medium replaces that spent using other mediators (Nie, Hillygus, \& Erbring, 2002; Shklovski, Kraut, \& Rainie, 2004), implying that online communication users have spent time in online settings rather than offline situations and then their subjective well-being would be harmed (Morgan \& Cotten, 2003; Nie, Hillygus, \& Erbring, 2002; Nie, 2001; Weiser, 2001). For example, Kraut et al. (1998) suggested that online communication users substitute weak ties for strong ones, implying that Internet motivates adolescents to form online contacts with strangers, instead of maintaining relationships with existing friends. The online contacts, which are characterized by superficial weak-tie relationships, are thought to reduce the quality of adolescents' existing friendships and their well-being.

The augmentation hypothesis holds that certain medium of communication can facilitate the use of others (Katz \& Rice, 2002; Turke, 1995). Specifically, the use of e-mail or Instant Messaging may improve face-to-face interaction and promote users' well-being (Morgan \& Cotten, 2003; Shaw \& Gant, 2002; Valkenburg \& Peter, 2007). For instance, using a sample of 1,210 Dutch teenagers between 10 and 17 years of age, Valkenburg and Peter (2007) found that Instant Messaging, which was mostly used to communicate with existing friends, is a significant predictor of users' subjective well-being.

Aside from studies supporting the augmentation or displacement hypothesis, there are also studies reporting no significant associations between online communication and subjective well-being (Gross, 2004; Jackson et al., 2004; Kraut, 2002; LaRose, Ghuay, \& Boivin, 2002; Mesch, 2001, 2003; Sanders, Field, Diego, \& Kaplan, 2000; Waestlund, Norlander, \& Archer, 2001). Current literature lack research data about online communication in China, especially in sample of adolescents. Our research studies Internet communication in a sample of Chinese adolescents with the goal of understanding the relationship between online communication and subjective well-being.

Earlier research suggested that online communication would reduce adolescents' social connectedness and well-being (Kraut et al., 1998). Researchers holding this idea believed that Internet motivates adolescents to form superficial online relationship with strangers, to spend more time with online strangers rather than friends in reality world, and then harm their interpersonal relationships and well-being (Kraut et al., 1998). This assumed relationship between online communication and quality of friendship were supported by some studies (Kraut et al., 1998). However, in recent studies, it has been found that, when users communicate with their existing friends, online communication can enhance the interaction between users and promote their well-being (Morgan \& Cotten, 2003; Shaw \& Gant, 2002; Val- 
kenburg \& Peter, 2007). It may be that, at the initial stage of online communication technology, users mainly use it for communication with strangers because of their curiosity on new emerging technology. Yet in recent years, it has been found that users mostly communicate with existing friends through Internet, which will promote their closeness to their friends and then improve their subjective well-being. Therefore, in our study, we put forward our first hypothesis that online communication will promote users' subjective well-being.

Compared with adolescent girls, adolescent boys tend to have a deeper and wider self-disclosure in online settings than in offline settings (Schouten, Valkenburg, \& Peter, 2007). Selfdisclosure is thought to be an important factor that has a positive influence on individuals' interpersonal relationship and well-being (Berndt, 2002). Adolescent boys usually face more inhibitions when disclosing themselves in offline settings than adolescent girls do. In online settings, where factors that might cause uneasiness in communication (e.g., audio/visual cues) are removed, adolescent boys will be more likely to disclose themselves better. Relevant studies have found that Internet use may have different effects on boys and girls. Using a sample of 85 Israeli university students, Amichai-Hamburger and Ben-Artzi (2003) found that the correlations between social, informational, leisure Internet use and loneliness were $.01, .001$, and -.28 for males, and .38, .18, and -.04 for females. Cooper (2003) obtained that the correlation coefficient between depression and Internet use was .47 for boys and .22 for girls. In the present study, we put forward our second hypothesis that online communication will have a bigger effect on subjective well-being for boys than for girls.

\section{Methods}

\section{Procedure and Participants}

The data were gathered in four classes of approximately 60 participants each. After obtaining informed consent, participants were given 45 minutes to complete the paper-and-pencil questionnaire. All participants were treated according to the ethical guidelines of the American Psychological Association (APA, 2001).

The participants were 190 adolescents ( 87 boys, 103 girls) from a small vocational school in the southwest area of China. Adolescents ranged in age from 15 to 19 years $(M=16.64, S D$ $=1.22$ ). $73 \%$ were from urban areas while $27 \%$ were from countryside areas. Approximately $83 \%$ were Han majority, while $9 \%$ were Tujia minority, $8 \%$ were Miao minority. These demographics represent the student population at this school.

\section{Measures}

\section{Online Communication}

We measured online communication with four adapted questions that have been used in Valkenburg and Peters' study (2006): a) "On weekdays (Monday to and including Friday), how many days do you usually use $\mathrm{QQ}^{1}$ ?" b) "On the weekdays (Monday to and including Friday) that you use QQ, how long

${ }^{1}$ To Tencent QQ, generally referred to as QQ, is the most popular free instant messaging computer program in Mainland China. As of September 30, 2010, the active QQ users' accounts for QQ IM amounted to 636.6 million, possibly making it the world's largest online community.
Table 1.

Descriptive data for online communication and subjective well-being.

\begin{tabular}{ccccc}
\hline Group & \multicolumn{2}{c}{ Online communication } & \multicolumn{2}{c}{ Subjective well-being } \\
\hline & $M$ & $S D$ & $M$ & $S D$ \\
\hline Overall & 12.30 & 10.50 & 3.52 & .79 \\
Boys & 11.40 & 9.83 & 4.75 & .85 \\
Girls & 13.07 & 11.03 & 3.68 & .71 \\
\hline
\end{tabular}

do you then usually use it?" c) "During weekends (Saturday and Sunday), how many days do you usually use QQ?" The response options were: 1) only on Saturday; 2) only on Sunday; 3) on both days; and 4) I do not use QQ on the weekends. If respondents selected response options 1 to 3 in the question on QQ weekend use, they were asked the following questions for Saturday and /or Sunday: d) "On a Saturday (Sunday), how long do you usually use QQ?" Respondents' QQ use per week was calculated by multiplying the number of days per week that they used QQ (range 0 through 7) by the number of minutes they used it on each day. This operationalization of weekly time spent with a medium has been proven valid for children older than 9 (Vander Voort \& Vooijs, 1990).

\section{Subjective Well-Being}

The Subjective Well-Being Scale is used in order to measure university students' subjective well-being. This scale developed by Tuzgöl-Dost (2005). Internal reliability for the Subjective Wel-Being Scale was a Cronbach-alfa coefficient of .93. Test re-test reliability yielded a correlation coefficient of $r=.86$ (Tuzgöl-Dost, 2006). In the present study, a Chinese edition of this scale was acquired through a translation and retranslation process. It has a Cronbach-alfa coefficient of .89 in the present study.

\section{Results}

The adolescents in the sample reported that they used online communication on average for 12.31 hours a week. No gender differences were found (males $=11.40 \pm 9.83$; females $=13.07$ \pm 11.03 hours/week; $t=1.09, p<.05)$ (please see Table 1 ). Most of the respondents $(67 \%)$ indicated that they mainly use QQ for communication with existing friends.

\section{Regression Coefficients of Online Communication on Subjective Well-Being}

A liner regression analysis was performed with the score on online communication as the independent variable and the subjective well-being score as the dependent variable. A significant model emerged $\left(F=11.986, p<.001, r^{2}=.060, \beta=.25\right)$ in which the online communication score was demonstrated to be a positive predictor of online self-disclosure.

\section{Coefficients of Determination of Online Communication on Subjective Well-Being among Girls and Boys}

To make a further understanding on the relationship between online communication and subjective well-being, another two linear regression analysis were performed to examine the pre- 
dictive effects of online communication on subjective wellbeing among girls and boys respectively. Following the recommendation of Cohen (1994), Rosenthal (1995) and Schmidt (1996), the coefficients of determination, namely $r^{2}$ values, will be used in interpreting the "research significance" or importance of the relationships. Specifically, a decision was made to only interpret as "meaningful" $r^{2}$ values that were greater than .050 . Thus, at least $5 \%$ of the online self-disclosure variance had to be explained for an effect to be interpreted as "important". There are a number of advantages to using the coefficient of determination in interpreting the value or importance of findings. First, use of this effect size index focuses attention on variance explained and hence helps researchers "honest" by reducing the temptation to inappropriately inflate or deflate the importance of findings. Cohen (1994) noted that these tendencies are sometimes all too common in studies that depend on interpretation of $p$ values alone. In addition, because $r^{2}$ serves as an index of relative strength, its use allows researchers to meaningfully compare and contrast effect sizes. For example, if an $r^{2}$ for the association between online communication and online self-disclosure is .10 for females and .05 for males, one can conclude that the magnitude of the relationship is twice as strong for females as compared to males. In the former case, $10 \%$ of the variance is accounted for, while in the latter case only $5 \%$ of the variance is accounted for.

In the present study, the results showed that, for boys, the coefficient of determination of online communication on subjective well-being was .143; while for girls, the regression coefficient of online communication on subjective well-being was .040. The coefficient of determination for boys was almost five times of that for girls, indicating that boys' online communication has a more important role on their subjective wellbeing than that for girls.

\section{Discussion}

In consistent with recent studies (Valkenburg \& Peter, 2007), the present study found that adolescents mainly use Internet Messaging for communication with existing friends. Participants reported that they spent an average of 12.31 hours last week on online communication, with $67 \%$ indicating that they use Internet to communicate with their existing friends. For boys, they spent an average of 11.40 hours on online communication while for girls they spent an average of 13.07 hours on it last week. No gender difference was found on the mean time of online communication $(t=1.09, p>.05)$.

The main purpose of this study was to examine the predictive effect of online communication on subjective with a sample of Chinese adolescents. In the present study, participants spending more time on online communication reported higher level of subjective well-being $\left(F=11.986, p<.001, r^{2}=.060, \beta=.25\right)$, a finding that has supported our first hypothesis and is consistent with previous studies which have found that online communication is positively related to users' well-being (Boneva, Quinn, Kraut et al., 2006; Coleman, Paternite, \& Sherman, 1999; Gross, Juvonen, \& Gable, 2002; Joinson, 2001; Tidwell $\&$ Walther, 2002). It may be that, online communication with existing friends can promote users' interaction in offline settings, which could strengthen their closeness to friends and improve their subjective well-being.
A further analysis reveals that boys can profit more from online communication than girls do, which has supported our second hypothesis. For boys the coefficient of determination was .143 and for girls .040. This finding is in keeping with previous relevant studies which have found that the correlation coefficient between Internet use and well-being were larger for boys than for girls (Copper, 2003). The finding that adolescent boys benefit more from online communication may be caused by the reason that adolescent boys tend to disclose themselves better than do adolescent girls in online settings. Self-disclosure has been proposed as an important factor influencing individuals' well-being. It has been found that respondents who reporting high level of self-disclosure tend to score higher on wellbeing measurement (Valkenburg \& Peter, 2007). Compared with adolescent girls, adolescent boys usually face more inhibittions when disclosing themselves in offline settings, and when they are in online settings, they can disclose themselves better because the reduced audiovisual cues make them feel less uneasiness. In this line, online communication has more impact on well-being for boys than for girls.

\section{Limitation}

This study is one of the first to explore predictive effects of online communication on users' subjective well-being with a sample that typically using Instant Message in China. Also this is one of the first to find that online communication has different extent of effects on subjective well-being for boys and girls. Despite the strengths, the results of this study should be viewed in light of some limitations. First, the sample used in this study is a relatively small and homogeneous group of students at a small vocational school. Therefore cautions should be made when the conclusions in this study are generalized to other populations. Second, the data were obtained from a cross-section design, which prevents the determination of casual relationships. Third, in this study, participants were asked to recall their online communication occurring last week, the memory bias is unavoidable. In the future study, researchers should consider using a qualitative approach aimed at complementing survey data, to better understand the association between online communication and subjective well-being. Additionally, experimental and longitudinal studies should be conducted to provide stronger evidence on the associations among variables examined in this study. Last, it should be noticed that it is not the case that online communication always has positive impact on users' subjective well-being. As researchers have suggested, online communication can have a positive influence on quality of friendship and well-being only when it is used for communication with existing friends (Bessière et al., 2008; Valkenburg $\&$ Peter, 2007). When online communication is used for conversation with strangers, however, the Internet users` friendship might be negatively impacted (Valkenburg \& Jochen, 2009).

\section{Conclusion}

1) Online communication with existing friends has a positive effect on adolescents' subjective well-being.

2) Online communication with existing friends has a bigger positive effect on subjective well-being for boys than for girls. 


\section{Acknowledgements}

The present study was supported by the Key Project 'Antecedents and Social Consequences of Online Communication among Adolescents' at Key Humanity Social Science Research Institute in Chongqing. Thank the two anonymous reviewers for their valuable suggestions on the manuscript.

\section{References}

Amichai-Hamburger, Y. \& Ben-Artzi, E. (2003). Loneliness and Internet use. Computers in Human Behavior, 19, 71-80. doi:10.1016/S0747-5632(02)00014-6

American Psychological Association (2001). Publication mannucal of the American Psychological Association (5th ed.). Washington, DC: Author.

Berndt, T. J. (2002). Friendship quality and social development. Current Directions in Psychological Science, 11, 7-10. doi:10.1111/1467-8721.00157

Bessière, K., Kiesler, S., Kraut, R., \& Boneva, B. S. (2008). Effects of Internet use and social resources on changes in depression. Information, Communication, and Society, 11, 47-70. doi:10.1080/13691180701858851

Boneva, B., Quinn, A., Kraut, R. et al. (2006). Teenage communication in the instant messaging era. In Computers, phones, and the Internet: domesticating information technology (pp. 201-218). New York: Oxford University Press.

Carden, R., \& Rettew, S. (2006). Internet chat room use, satisfaction with life, and loneliness. Psychological Reports, 98, 121-122. doi:10.2466/pr0.98.1.121-122

Cohen, J. (1994). The earth is round $(p<0.05)$. American Psychologist, 49, 997-1003. doi:10.1037/0003-066X.49.12.997

Coleman, L. H., Paternite, C. E., \& Sherman, R. C. (1999). A reexamination of deindividuation in synchronous computer-mediated communication. Computers in Human Behavior, 15, 51-65. doi:10.1016/S0747-5632(98)00032-6

Cooper, N. S. (2003). The identification of psychological and social correlates of Internet use in children and teenagers. Ph. D. Thesis, Los Angeles, California: Alliant International University.

Gross, E. F. (2004). Adolescent Internet use: What we expect, what teens report. Journal of Applied Developmental Psychology, 25, 633-649. doi:10.1016/j.appdev.2004.09.005

Gross, E., Juvonen, J., \& Gable, S. (2002). Internet use and well-being in adolescence. Journal of Social Issues, 58, 75-90. doi:10.1111/1540-4560.00249

Jackson, L. A., Von Eye, A., Barbatsis, G., Biocca, F., Fitzgerald, H. E., $\&$ Zhao, Y. (2004). The impact of Internet use on the other side of the digital divide. Communications of the ACM, 47, 43-47. doi:10.1145/1005817.1005819

Joinson, A. (2001). Self-disclosure in computer-mediated communication: The role of self-awareness and visual anonymity. European Journal of Social Psychology, 31, 177-192.

doi:10.1002/ejsp.36

Katz, J. E., \& Rice, R. E. (2002). Syntopia: access, civic involvement, and social interaction on the Net. In C. Haythornthwaite and B. Wellman (Ed.), The Internet in everyday life (pp. 114-138). Malden, MA: Blackwell.

Kraut, R., Kiesler, S., Boneva, B., Cummings, J., Helgeson, V., \& Crawford, A. (2002). Internet paradox revisited. Journal of Social Issues, 58, 49-74. doi:10.1111/1540-4560.00248

Kraut, R., Patterson, M., Lundmark, V., Kiesler, S., Mukopadhyay, T., \& Scherlis, W. (1998). Internet paradox: A social technology that reduces social involvement and psychological well-being? American Psychologist, 53, 1017-1031. doi:10.1037/0003-066X.53.9.1017

LaRose, S., Ghuay, F., \& Boivin, M. (2002). Attachment, social support, and loneliness in young adulthood: A test of two models. Personality and Social Psychology Bulletin, 28, 684-693. doi: $10.1177 / 0146167202288012$

Mesch, G. (2001). Social relationships and Internet use among adolescents in Israel. Social Science Quarterly, 82, 329-339. doi:10.1111/0038-4941.00026

Mesch, G. (2003). The family and the Internet: The Israeli case. Social Science Quarterly, 84, 1050-1083. doi:10.1046/j.0038-4941.2003.08404016.x

Morgan, C., \& Cotten, S. R. (2003). The relationship between Internet activities and depressive symptoms in a sample of college freshmen. Cyberpsychology \& Behavior, 6, 133-141. doi:10.1089/109493103321640329

Nie, N. H, Hillygus D. S, \& Erbring L. (2002). Internet use, interpersonal relations, and sociability. In B. Wellman and C. Haythornthwaite (Eds.), The Internet in everyday life (pp. 215-243). Malden, MA: Blackwell. doi:10.1002/9780470774298.ch7

Nie, N. H. (2001). Sociability, interpersonal relations and the Internet: Reconciling conflicting findings. American Behavioral Scientist. American Behavioral Scientist, 45, 420-435. doi: $10.1177 / 00027640121957277$

Rosenthal, R. (1995). Progress in clinical psychology: Is there any? Clinical Psychology: Science and Practice, 2, 133-149. doi:10.1111/j.1468-2850.1995.tb00035.x

Sanders, C. E., Field, T. M., Diego, M., \& Kaplan, M. (2000). The relationship of Internet use to depression and social isolation. Adolescence, 35, 237-242.

Schouten, A. P., Valkenburg, P. M., \& Peter, J. (2007). Precursors and underlying processes of adolescents' online self-disclosure: Developing and testing an "Internet-attribute-perception" model. Media Psychology, 10, 292-314. doi:10.1080/15213260701375686

Schiffrin, H., Edelman, A., Falkenstern, M., \& Stewart, C. (2010). The associations among computer-mediated communication, relationships, and well-being. Cyberpsychology Behavior and Social Networking, 3, 299-306. doi:10.1089/cyber.2009.0173

Schmidt, F. (1996). Statistical significance testing and cumulative knowledge in psychology: Implications for training of researchers. Psychological Methods, 1,115-129. doi:10.1037/1082-989X.1.2.115

Shaw, L. H., \& Gant, L. M. (2002). In defense of the Internet: The relationship between Internet communication and depression, loneliness, self-esteem, and perceived social support. CyberPsychology \& Behavior, 5, 157-170. doi:10.1089/109493102753770552

Shklovski, I., Kraut R, \& Rainie L. (2004). The Internet and social participation: contrasting cross-sectional and longitudinal analyses. Journal of Computer-Mediated Communication, 10, Article 1.

Tidwell, L., \& Walther, J. (2002). Computer-mediated communication effects on disclosure, impressions, and interpersonal evaluations: getting to know one another a bit at a time. Human Communication Research, 28, 317-348. doi:10.1111/j.1468-2958.2002.tb00811.x

Turke, S. (1995). Life on the screen: identity in the age of the Internet. New York: Simon \& Schuster.

Tuzgöl Dost, M. (2005). Öznel øyi Oluú Ölçe-i’nin geliútirilmesi: Geçerlik ve güvenirlik çalÕúmasÕ. Türk Psikolojik DanÕúma ve Rehberlik Dergisi, 3, 103-109.

Tuzgöl-Dost, M. (2006). SubjectÕve well-beÕng among unÕversÕty students. Hacettepe Üniversitesi E:itim Fakültesi Dergisi, 31, 188-197.

Valkenburg, P. W., \& Peter, J. (2007). Online communication and adolescent well-being: Testing the stimulation versus the displacement hypothesis. Journal of Computer-Mediated Communication, 12, 1169-1182. doi:10.1111/j.1083-6101.2007.00368.x

Valkenburg, P. M., \& Peter, J. (2009). Social consequences of the Internet for adolescents: A decade of research. Current Directions in Psychological Science (Wiley-Blackwell), 18, 1-5.

Waestlund, E., Norlander, T., \& Archer, T. (2001). Internet blues revisited: Replication and extension of an Internet paradox study. Cyberpsychology \& Behavior, 4, 385-391. doi: $10.1089 / 109493101300210295$

Weiser, E. B. (2001). The functions of Internet use and their psychological consequences. Cyberpsychology \& Behavior, 4, 723-744. doi: $10.1089 / 109493101753376678$ 